\title{
Special Issue "Kinetic Theory and Swarming Tools to Modeling Complex Systems-Symmetry problems in the Science of Living Systems"-Editorial and Research Perspectives
}

\author{
Nicola Bellomo ${ }^{1,2,3, *(\mathbb{D})}$, Damián A. Knopoff ${ }^{4}$ (D) and Pietro Terna ${ }^{5}$ (D) \\ 1 Departamento de Matemática Aplicada, University of Granada, 18071 Granada, Spain \\ 2 Department of Mathematical Sciences, Politecnico di Torino, 10100 Torino, Italy \\ 3 IMATI CNR, 27100 Pavia, Italy \\ 4 CIEM CONICET and FaMAF-National University of Cordoba, 5000 Cordoba, Argentina; \\ damian.knopoff@unc.edu.ar \\ 5 School of Management and Economics, University of Torin, 10100 Torino, Italy; pietro.terna@unito.it \\ * Correspondence: nicola.bellomo@polito.it
}

Received: 4 March 2020; Accepted: 9 March 2020; Published: 13 March 2020

check for updates

\begin{abstract}
This editorial paper presents a special issue devoted to the development of mathematical tools from kinetic and swarms theory to the modeling and simulations of the dynamics of living systems constituted by very many interacting living entities. Applications refer to several fields: collective learning, behavioral economy, multicellular systems, vehicular traffic, and human crowds. A forward look to research perspectives is focused on the conceptual links between swarms methods and the kinetic theory approach.
\end{abstract}

Keywords: kinetic theory; living systems; social dynamics; active particles; learning; social dynamics; pattern formation

\section{Introduction}

This paper presents the conceptual framework of the special issue "Kinetic Theory and Swarming Tools to Modeling Complex Systems-Symmetry problems in the Science of Living Systems" published in the journal Symmetry. The special issue is devoted to report the research activity in the field by means of a selection of scientific articles, where mathematical tools of the kinetic theory and swarms dynamics can contribute to modeling and simulations of living systems. Indeed, it focuses on a fascinating objective which cannot be tackled by the approach of the so-called hard sciences, specifically mathematics without the invention of new mathematical theories.

It is a highly challenging objective. We cannot naively claim that the contents of this issue provide fundamental contributions to the said objective. On the other hand, it can be claimed that the contents witness the ongoing research activity as well as the growing interest of scientists this field requires an interdisciplinary approach.

Essentially, all papers published in the issue technically refer to the so called kinetic theory of active particles, which is a mathematical theory towards the modeling of large systems of interacting living entities [1]. The edited collection of surveys [2] reports about a broad variety of fields of applications from social sciences to the dynamics of vehicular traffic. The key problem of the modeling approach consists in capturing the complexity features of living systems.

Applications have been developed in various fields of life sciences and, more in general, the so-called behavioral sciences where individual behaviors play a key role in the interactions 
and subsequently on the overall dynamics. The survey [3] shows how research activity keeps attracting a growing interest of applied mathematicians and physicists. The very first step of the methodological approach is the representation of the system by a distribution function which defines the probability distribution over the state at the micro scale (individual based) of the interacting entities. Subsequently, the modeling of interactions can be developed according to the specific features of the specific system under consideration, and finally tools of statistical physics are developed to transfer the dynamics of interactions to that of the collective behaviors of the whole system.

It is worth stressing that the specific features of living systems often lead to nonsymmetric interactions, namely, loss of symmetry appears in the dynamics at the low scale. In fact, living entities always apply a selection to the various signals received in the interaction with other entities.

The interested reader can refer to [1] to obtain a picture of the details and of the framework which constitutes the conceptual basis of the mathematical theory. Therefore, we do not add here further details on the methodological approach, but simply mention that interactions present key features which are typical of living systems, namely interactions are nonlocal and nonlinearly additive, collective and often non symmetric. These features are shared also by alternative approaches such as the theory of swarms reviewed in [4] and developed after the celebrated paper by Cucker and Smale [5], while the interest of applied mathematicians and physicists is witnessed in vast literature, e.g., [6-8] including papers specifically related to economic problems $[9,10]$. In addition, we mention with the aim of providing a fully detailed framework the kinetic theory approach developed by mean field and Fokker-Plank models [11], where a variety of interesting models mainly on social dynamics are reported and implemented by sharp numerical tools.

This editorial note is not limited to report about the contents, but it aims also to develop a forward look to research perspectives somehow motivated by the contents of the issue. The presentation of the contents given in Section 2 is followed by a forward look to research perspectives which is proposed in Section 3. In more details, the outline of the research perspectives avoids being generic and focuses on a well defined topic, namely the link between swarms and kinetic approaches somehow related to the development by discrete variables methods. This topic is treated within a multiscale vision somehow inspired by the sixth Hilbert problem [12].

\section{On the Contents of the Special Issue}

Let us now present the contents of the issue following a sequence somehow related to the rationale of the modeling approach, where each paper is related to the conceptual framework and some key literature related to it.

- A new approach to collective learning is proposed in [13] following previous contributions of the authors, where they developed an approach based on the kinetic theory of active particles [14,15]. The novel contents proposed in [13] refer to the specialization of the different types of learning, which is proposed in the fist part of the paper, and the study of the interactions between collective learning and different types of dynamics, which appears in almost all behavioral phenomena, for instance social conflicts related to welfare strategies [16,17]. Learning dynamics is a key feature of the various dynamics treated in the papers cited in the following.

- A model of opinion dynamics is studied in [18], where a sharp asymptotic analysis shows how kinetic type models lead to diffusion problems. This paper refers to a topic which has been widely studied by the kinetic theory approach, for instance [19-21], where learning dynamics is the first step of the complex process leading to opinion formation.

- A contribution to behavioral economy is given in [22]. The authors specifically refer to the approach of the kinetic theory for active particles [23-25] which appears to be effective in capturing the main features of behavioral economy [26,27]. Heterogeneity, up to unethical behaviors [28-30], and interactions between economy and social sciences are fundamental aspects of the mathematical approach to behavioral economy. 
- The biology of cells, in particular the immune competition, has been one of the very first fields of application of kinetic theory methods [31]. Motivations to account for the specific features of cells, to be viewed as a living system, have been frequently posed to mathematicians and physicists by biologists, as shown by the celebrated paper by the Nobel Laureate Leland Hartwell [32] who, focusing on biological systems, indicates some important features which distinguish living systems from the inert matter. Indeed, research hints look at a new biology for this century [33]. The dynamics of cell motion is treated in [34], where authors account for structure of the extracellular matrix, considering cell membrane reactions, haptotaxis and chemotaxis. The modeling is performed at a microscopic scale, while a macroscopic model is derived by a scaling limit.

- The kinetic theory approach to vehicular traffic was initiated by the visionary idea of Prigogine [35]. An interesting contribution to our special issue has been delivered in [36] for models where the microscopic state includes, in addition to position and velocity, also an additional variable deemed to describe the quality of the driver-vehicle micro-system. An additional novelty of this paper is that both short-range and mean field interactions are introduced to depict velocity changes related to passing phenomena in view of modeling the role of toll gates or traffic highlights.

- Two papers have been published on modeling and simulation of the dynamics of human crowds. The first one [37] motivates the kinetic theory approach as the most appropriate scale to describe the dynamics of human crowds. Indeed, the authors show that models at this scale have the ability to capture several features of human crowds, for instance subdivision into different groups pursuing different walking strategies, heterogeneous distribution of the walking ability, interaction between emotional states and walking strategies. The second paper [38], in turn, tackles the problem of simulating the dynamics of human crowds under stress conditions in venues with internal obstacles. Applied mathematicians have devoted a great deal of energy to this research topics which has an impact on safety problems and require advanced mathematical tools as witnessed in the very recent literature, see $[4,11,39-43]$. The authors account for the pertinent literature and develop simulations in a geometry somehow inspired to that of Jamarat bridge. Montecarlo particle methods [44,45] have been used to develop simulations. The application of this computational approach is not straightforward due to the presence of the activity variable, on the other hand it is the most appropriate to account for the specific stochastic feature of kinetic models.

\section{On a Forward Look to Research Perspectives}

The interesting contributions to this special issue motivate some reasonings on research perspectives. However, rather than producing a list of open problems, we will focus this section on a specific research perspective, namely, a multiscale vision of living systems. This topic has been selected, out of various possible ones, according to the idea that only multiscale methods can lead to a consistent description of living systems. Indeed, this is the visionary concept, posed to the attention of mathematicians and physicists, in the Sixth Hilbert problem [12].

The reference scale, which this special issue refers to, is the mesoscale based on kinetic theory methods which has been applied by all the papers herein reviewed. It is possible, according to the methodological approach proposed in [46,47], to derive models at the macroscopic scale from the underlying description at the microscale. Some examples are available in the literature concerning vehicular traffic [48], crowd dynamics [49], and multicellular systems [50,51].

On the other hand, a key problem, related to the selection of the representation and modeling scale, is the validity of the continuity assumption of the probability distribution. Indeed, this assumption is consistent only if the number of interacting a-particles is high enough. Arguably, the number of interacting entities is not large enough to justify the aforementioned assumption. Hence, a research perspective consists in looking for dynamical systems with finite degrees of freedom suitable to model systems at the lower scale. 
This objective requires the derivation of a mathematical framework accounting for the specific features of the interaction dynamics, namely nonlinear additivity and nonlocal dynamics. A first step towards this specific objective has been proposed in [52], referring to a large system of active particles subdivided into functional subsystems. In more detail, the following general framework has been proposed:

$$
\left\{\begin{array}{l}
\frac{d u_{i j}}{d t}=v_{i j}, \\
\frac{d v_{i j}}{d t}=\sum_{p=1}^{n} \sum_{q=1}^{N_{p}} \eta_{i j}^{p q}(\mathbf{u}, \mathbf{v}) \varphi_{i j}^{p q}(\mathbf{u}, \mathbf{v})+\sum_{p=1}^{n} \mu_{i j}^{p}\left(\mathbf{u}, \mathbb{E}_{p}\right) \psi_{i j}^{p}\left(\mathbf{u}, \mathbb{E}_{p}\right),
\end{array}\right.
$$

where the subscript $i, j$ identify, respectively, the $i$-th functional subsystem and the $j$-th a-particle, while $N_{i}$ denotes the number of particles in the i-th FS, and $n$ the number of FSs. In addition:

$\eta_{i j}^{p q}$ models the interaction rate of individual based interactions between $i j$-particles and $p q$-particles; $\mu_{i j}^{p}$ models the micro-macro interaction rate between $i j$-particles and $p$-functional subsystem; $\varphi_{i j}^{p q}$ denotes the micro-micro action, which occurs with rate $\eta_{i j}^{p q}$, of an $p q$-particle over an $i j$-particle; $\psi_{i j}^{p}$ denotes the micro-macro action, which occurs with rate $\mu_{i j}^{p}$ of a $p$-functional subsystem over an $i j$-particle.

The mathematical structure is obtained modeling the action given by the product $\eta \varphi$ for the micro-micro interactions and by $\mu \psi$ for the micro-macro interactions and by equating the overall action to the acceleration of the variable $u_{i j}$, where $\mathbf{u}=\mathbf{u}(t)$ and $\mathbf{v}=\mathbf{v}(t)$ denote, respectively, the set of all states $u_{i j}$ and speed of growth $v_{i j}$. Mathematical models are obtained by implementing the structure (1) with the description of individual based interactions. The application studied in [52] refers to modeling the dynamics of prices due to the interactions between producers-sellers and buyers.

It is useful observing that the behavioral swarm method can be viewed as a generalized mathematical formalization of the Agent-Based Model (ABM) field. In an ABM perspective [53-55] we have simple of complicated agents, with internal rules, interacting one to one or in groups or with institutions. The starting point is always a population of agents, representing individuals or more generally entities, as the component of a generic system, that we construct using small parts of computer code operating in dedicated software environments. The goal is to search for regularities at the macro level generated by the behavior of the agents (micro level, if individuals; meso level, if more complex entities).

In ABMs, we are close to swarms, but operating in a very different manner. The mathematical generalization requires limitations. As a consequence, we can develop our research in a double way, with both a swarm construction and an agent-based one. The first one can be guided by strict formal limitations, requiring to have a more in-depth look at the way the agents behave; the second one offers interesting verifications that we can obtain relaxing some of the constraints introduced in the swarm model. However, the behavioral swarm method provides a more general and flexible approach worth to be further developed towards applications in behavioral sciences [3].

Funding: This research received no external funding.

Conflicts of Interest: The authors declare no conflict of interest.

\section{References}

1. Bellomo, N.; Bellouquid, A.; Gibelli, L.; Outada, N. A Quest Towards a Mathematical Theory of Living Systems; Birkhäuser: New York, NY, USA, 2017.

2. Ball, P. Why Society is a Complex Matter; Springer: Heidelberg, Germany, 2012. 
3. Kwon, H.R.; Silva, E.A. Mapping the Landscape of Behavioral Theories: Systematic Literature Review. J. Plan. Lit. 2019. [CrossRef]

4. Albi, G.; Bellomo, N.; Fermo, L.; Ha, S.-Y.; Kim, J.; Pareschi, L.; Poyato, D.; Soler, J. Traffic, crowds, and swarms. From kinetic theory and multiscale methods to applications and research perspectives. Math. Model. Methods Appl. Sci. 2019, 29, 1901-2005. [CrossRef]

5. Cucker, F; Smale, S. Emergent behavior in flocks. IEEE Trans. Automat. Contr. 2007, 52, 853-862.

6. Bellomo, N.; Ha, S.-Y. A quest toward a mathematical theory of the dynamics of swarms. Math. Model. Methods Appl. Sci. 2017, 27, 745-770. [CrossRef]

7. Ha, S.-Y.; Kim, J.; Ruggeri, T. Emergent behaviors of thermodynamic Cucker-Smale particles. SIAM J. Math. Anal. 2018, 50, 3092-3121. [CrossRef]

8. Fang, D.; Ha, S.-Y.; Jin, S. Emergent behaviors of the Cucker-Smale ensemble under attractive-repulsive couplings and Rayleigh frictions. Math. Model. Methods Appl. Sci. 2019, 19, 1349-1385. [CrossRef]

9. Ahn, S.-M.; Bae, H.-O.; Seung, S.-Y.; Kim, Y.; Lim, H. Application of flocking mechanisms, to the modeling of stochastic volatily. Math. Models Methods Appl. Sci. 2013, 23, 1603-1628. [CrossRef]

10. Bae, H.-O.; Cho, S.-Y.; Kim, J.; Yun, S.-B. A kinetic description for the herding behavior in financial market. J. Stat. Phys. 2019, 176, 398-424. [CrossRef]

11. Pareschi, L.; Toscani, G. Interacting Multiagent Systems: Kinetic Equations and Monte Carlo Methods; Oxford University Press: Oxford, UK, 2013.

12. Hilbert, D. Mathematical problems. Bull. Am. Math. Soc. 1902, 8, 437-479. [CrossRef]

13. Burini, D.; De Lillo, S. On the complex interaction between collective learning and social dynamics. Symmetry 2019, 11, 967. [CrossRef]

14. Burini, D.; De Lillo, S.; Gibelli, L. Collective learning modeling based on the kinetic theory of active particles. Phys. Life Rev. 2016, 16, 126-139. [CrossRef] [PubMed]

15. Burini, D.; Gibelli, L.; Outada, N. A kinetic theory approach to the modeling of complex living systems. In Active Particles, Volume 1; Series: Modelling Simulations Science Engineering Technology; Springer: Berlin, Germany, 2017; pp. 229-258.

16. Bellomo, N.; Herrero, M.A.; Tosin, A. On the dynamics of social conflicts looking for the Black Swan. Kinet. Relat. Models 2013, 6, 459-479. [CrossRef]

17. Furioli, G.; Pulvirenti, A.; Terraneo, E.; Toscani, G. Fokker-Planck equations in the modeling of socio-economic phenomena. Math. Mod. Meth. Appl. Sci. 2017, 27, 115-158. [CrossRef]

18. Lachowicz, M.; Leszczyński, H.; Puźniakowska-Galuch, E. Diffusive and anti-diffusive behavior for kinetic models of opinion dynamics. Symmetry 2019, 11, 1024. [CrossRef]

19. Dolfin, D.; Lachowicz, M. Modeling opinion dynamics: How the network enhances consensus. Netw. Heterog. Media 2015, 4, 877-896. [CrossRef]

20. Knopoff, D. On the modeling of migration phenomena on small networks. Math. Mod. Meth. Appl. Sci. 2013, 23, 541-563. [CrossRef]

21. Knopoff, D. On a mathematical theory of complex systems on networks with application to opinion formation. Math. Model. Methods Appl. Sci. 2014, 24, 405-426. [CrossRef]

22. Dolfin, M.; Leonida, L.; Muzzupappa, E. Forecasting Efficient Risk/Return Frontier for Equity Risk with a KTAP Approach: Case Study in Milan Stock Exchange. Symmetry 2019, 11, 1055. [CrossRef]

23. Ajmone Marsan, G.; Bellomo, N.; Gibelli, L. Stochastic evolutionary differential games toward a systems theory of behavioral social dynamics. Math. Model. Methods Appl. Sci. 2016, 26, 1051-1093. [CrossRef]

24. Bellomo, N.; Colasuonno, F.; Knopoff, D.; Soler, J. From a systems theory of sociology to modeling the onset and evolution of criminality. Netw. Heterog. Media 2015, 10, 421-441. [CrossRef]

25. Dolfin, M.; Knopoff, D.; Leonida, L.; Patti, D. Escaping the trap of "blocking": A kinetic model linking economic development and political competition. Kinet. Relat. Model. 2017, 10, 423-443. [CrossRef]

26. Thaler, R.H.; Sunstein, C. Nudge: Improving Decisions About Health, Wealth, and Happiness; Penguin: New York, NY, USA, 2016.

27. Thaler, R.H. Behavioral Economics: Past, Present, and Future. Am. Econ. Rev. 2016, 106, 1577-1600. [CrossRef]

28. Piff, P.K.; Stancato, D.M.; Coté, S.; Mendoza-Denton, R.; Keltner, D. Higher social class predicts increased unethical behavior. Proc. Natl. Acad. Sci. USA 2014, 109, 4086-4091. [CrossRef] [PubMed] 
29. Salvi, S. Corruption corrupts: Society-level rule violations affect individuals' intrinsic honesty. Nature 2016, 531, 456-457. [CrossRef] [PubMed]

30. Liu, L.; Chen, X.; Szolnoki, A. Evolutionary dynamics of cooperation in a population with probabilistic corrupt enforcers and violators. Math. Model. Methods Appl. Sci. 2018, 29, 2127-2149. [CrossRef]

31. Bellouquid, A.; Delitala, M. Modelling Complex Biological Systems-A Kinetic Theory Approach. In Modeling and Simulation in Science, Engineering and Technology; Birkhäuser: Boston, MA, USA, 2006.

32. Hartwell, H.L.; Hopfield, J.J.; Leibler, S.; Murray, A.W. From molecular to modular cell biology. Nature 1999, 402, c47-c52. [CrossRef]

33. Woese, C.R. A new biology for a new century. Microbiol. Mol. Biol. Rev. 2004, 68, 173-186. [CrossRef]

34. Knopoff, D.; Nieto, J.; Urrutia, L. Numerical simulation of a multiscale cell motility model based on the kinetic theory of active particles. Symmetry 2019, 11, 1003. [CrossRef]

35. Prigogine, I.; Herman, R. Kinetic Theory of Vehicular Traffic; Elsevier: New York, NY, USA, 1971.

36. Calvo, J.; Nieto, J.; Zagour, M. Kinetic Model for Vehicular Traffic with Continuum Velocity and Mean Field Interactions. Symmetry 2019, 11, 1093. [CrossRef]

37. Elaiw, A.; Al-Turki, Y.; Alghamdi, M. A critical analysis of behavioural crowd dynamics: From a modelling strategy to kinetic theory methods. Symmetry 2019, 11, 851. [CrossRef]

38. Elaiw, A.; Al-Turki, Y. Particle methods simulations by kinetic theory models of human crowds accounting for stress conditions. Symmetry 2020, 12, 14. [CrossRef]

39. Aylaj, B.; Bellomo, N.; Gibelli, L.; Reali, A. On a unified multiscale vision of behavioral crowds. Math. Model. Methods Appl. Sci. 2020, 30, 1-22. [CrossRef]

40. Bellomo, N.; Gibelli, L.; Outada, N. On the interplay between behavioral dynamics and social interactions in human crowds. Kinet. Relat. Model. 2019, 12, 397-409. [CrossRef]

41. Bailo, R.; Carrillo, J.A.; Degond, P. Pedestrian models based on rational behaviour. In Crowd Dynamics, Volume 1-Theory, Models, and Safety Problems; Modeling and Simulation in Science, Engineering, and Technology; Birkhäuser: New York, NY, USA, 2018.

42. Goldsztein, G.H. Self-Organization When Pedestrians Move in Opposite Directions. Multi-Lane Circular Track Model. Appl. Sci. 2020, 10, 563. [CrossRef]

43. Kim, D.; Quaini, A. A kinetic theory approach to model pedestrian dynamics in bounded domains with obstacles. Kinet. Relat. Model. 2019, 12, 1273-1296. [CrossRef]

44. Aristov, V.V. Biological systems as nonequilibrium structures described by kinetic methods. Results Phys. 2019, 13, 102232. [CrossRef]

45. Barbante, P.; Frezzotti, A.; Gibelli, L. A kinetic theory description of liquid menisci at the microscale. Kinet. Relat. Model. 2015, 8, 235-254.

46. Burini, D.; Chouhad, N. Hilbert method toward a multiscale analysis from kinetic to macroscopic models for active particles. Math. Model. Methods Appl. Sci. 2017, 27, 1327-1353. [CrossRef]

47. Burini, D.; Chouhad, N. A Multiscale view of nonlinear diffusion in biology: From cells to tissues. Math. Model. Methods Appl. Sci. 2019, 29, 791-823. [CrossRef]

48. Bellomo, N.; Bellouquid, A.; Nieto, J.; Soler, J. On the multiscale modeling of vehicular traffic: From kinetic to hydrodynamics. Discret. Cont. Dyn. B 2014, 19, 1869-1888. [CrossRef]

49. Bellomo, N.; Bellouquid, A. On multiscale models of pedestrian crowds from mesoscopic to macroscopic. Commun. Math. Sci. 2015, 13, 1649-1664. [CrossRef]

50. Bellomo, N.; Bellouquid, A.; Nieto, J.; Soler, J. On the asymptotic theory from microscopic to macroscopic growing tissue models: An overview with perspectives. Math. Model. Methods Appl. Sci. 2012, 22. [CrossRef]

51. Bellomo, N.; Bellouquid, A.; Chouhad, N. From a multiscale derivation of nonlinear cross-diffusion models to Keller-Segel models in a Navier-Stokes fluid. Math. Model. Methods Appl. Sci. 2016, 26, 2041-2069. [CrossRef]

52. Bellomo, N.; De Nigris, S.; Knopoff, D.; Morini, M.; Terna, P. Swarms dynamics towards a systems approach to social sciences and behavioral economy. Netw. Heterog. Media 2020, in press.

53. Gilbert, N.; Terna, P. How to build and use agent-based models in social science. Mind Soc. 2000, 1, 57-72. [CrossRef] 
54. Tesfatsion, L. Agent-based computational economics: Modeling economies as complex adaptive systems. Inf. Sci. 2003, 149, 262-268. [CrossRef]

55. Grimm, V.; Railsback, S.F.; Vincenot, C.E.; Berger, U.; Gallagher, C.; DeAngelis, D.L.; Edmonds, B.; Ge, J.; Giske, J.; Groeneveld, J.; et al. The odd protocol for describing agent-based and other simulation models: A second update to improve clarity, replication, and structural realism. J. Artif. Soc. Simul. 2020, 23, 7.

(C) 2020 by the authors. Licensee MDPI, Basel, Switzerland. This article is an open access article distributed under the terms and conditions of the Creative Commons Attribution (CC BY) license (http:/ / creativecommons.org/licenses/by/4.0/). 